\title{
Modeling an Emergency Service System for a Hospital Network
}

\author{
Alain Wegmann ${ }^{1}$, Gil Regev ${ }^{1}$, Gilles Viotti ${ }^{2}$, Pierre-Alain Pilet $^{2}$, Philippe Vuagniaux $^{2}$ \\ ${ }^{\prime}$ Ecole Polytechnique Fédérale de Lausanne (EPFL), \\ School of Computer and Communication Sciences, CH-1015 Lausanne, Switzerland, \\ \{alain.wegmann, gil.regev\}@epfl.ch \\ 2FHV Informatique, CH-1008 Prilly, Switzerland
}

\begin{abstract}
In this paper we describe a service modeling project done at an IT department that provides IT services to a network of state owned healthcare providers in the state of Vaud in Switzerland. The goal of the project is to understand how to maintain business continuity in the case of a disaster affecting the IT department's data center. We analyze how to precisely relate the business requirements to the IT operation requirements with the help of the SEAM Enterprise Architecture method. The results are refined service levels and the identification of the required technical architecture.
\end{abstract}

Keywords: Service System, Business Continuity, Service Modeling, IT Infrastructure, Healthcare, SEAM

\section{Introduction}

FHV (www.fhv.ch) is the organization that coordinates most regional hospitals in the state of Vaud, Switzerland. It includes 12 hospitals (among them: hospitals providing emergency services, psychiatric hospitals, and hospitals specialized in re-adaption and in convalescence). In 2010, FHV provided 600K days of care.

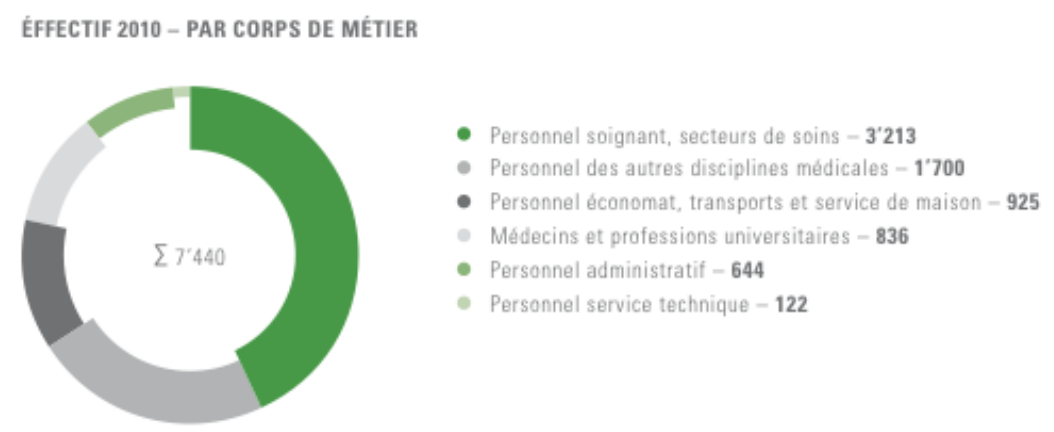

Figure 1 FHV Employee Distribution 
FHVi (www.fhvi.ch) is the internal IT provider of FHV. FHVi manages a centrally located data center that provides its services across the state of Vaud. FHVi also manages the IT infrastructures located in the 12 hospitals, spread all around the state. FHVi has about 50 employees (25 in application management and 25 in IT infrastructure and support). They manage 300 servers (physical and virtual) and 3500 workstations spread in the FHV hospitals. FHVi provides 80 IT services to the hospitals.

FHVi works with external vendors that supply it with IT applications; these vendors provide executable code, documentation and, sometimes, IT services. Application managers are responsible to understand the user requirements, to manage the developments made by these vendors and to define a strategy for the application or service under their responsibility. Infrastructure managers are responsible for the execution, storage and communication infrastructure that host the applications. Infrastructure managers can specialize in a specific kind of infrastructure category e.g. virtual machines, storage, or network.

In their risk analysis, FHVi identified that their single data center was a significant risk. If data center were to be damaged (by e.g., fire or flood), the services to the hospitals would be impacted very significantly for a very long period of time (months). For this reason, FHVi started a project for creating a backup data center located somewhere else in the state. The project was named psico ("plan secours informatique et de continuité").

Prior to the psico project, FHVi already defined their IT service catalog. As a result, they identified 4 IT services that they provide, which are considered to be critical to the functioning of the FHV hospitals. These IT services must be provided even if the data center is damaged. These services are: medical imaging, patient medical management, patient administrative management, and laboratory management. The medical imaging service is supported by two applications: image processing application and radiology records application.

FHVi defined three operational modes in connection with the risk of damage to the main data center:

- Normal mode - The data center runs properly. The hospitals can rely on its services for the services they provide to their stakeholders.

- Disconnected mode - This is a degraded mode that immediately follows an incident that brings down the data center. In this mode, the hospital is not connected to the data center anymore but can run autonomously (i.e. the hospital's local infrastructure is not affected by the incident). For, example, if the data center is down, hospitals can still do radiology exams and look up for images in a one-year archive. In the disconnected mode, the hospital's medical imaging systems run stand-alone. This means that they are not connected to the patient administrative management (so information has to be keyed in by hand in the imaging device before doing an exam) or to the radiology records application (so no radiology diagnostics record can be entered or accessed). In the negotiation between hospitals and FHVi, this level of service was considered as acceptable for 24 hours but not for longer.

- Psico mode - In this mode the four critical IT services are provided in whole or part. For the medical imaging service, both applications (images and records) must be available. The hospitals can work for weeks in this 
mode. The FHVi is in psico mode as soon as the backup data center takes over from the downed main data center. In this mode, the hospitals' systems are not disconnected anymore but are connected to the backup data center.

Initially, the psico project encompassed two subprojects: The backup site selection and equipment project and the IT Service Continuity (ITSCM) project. The initial goals of the ITSCM project were

1. To bring together application managers with infrastructure managers so as to agree on a common understanding of the complete service delivered by the IT.

2. To define the requirements for the reduced operation mode for the critical services described above. More specifically, in which sequence the IT systems are to be resumed when the FHVi will switch from the main data center to the backup data center.

3. To define the architecture necessary to support the requirements.

In this project, we did not analyze other risks (such as a disaster affecting the hospitals). These risks are analyzed in the standard risk management process of FHV and FHVi.

Following a presentation of the SEAM Enterprise Architecture method $[2,3,13,16,17]$ given by the first author in an industry conference, FHVi invited him to help with the ITSCM project. A core concept of SEAM is the modeling and alignment of services. The services provided by an organization to its stakeholders are modeled and aligned with the services provided by the IT to stakeholders. SEAM was therefore found to be suitable for the ITSCM project.

The ITSCM project was divided in two parts: a first series of 3 workshops was planned before the official kick-off of the project with the goal of scoping the project. A second series of 4 workshops was planned; workshops in which the business requirements were refined, the existing architecture was modeled, problems with the existing infrastructure were identified and the architecture of a solution was drafted. All workshops lasted half a day each and brought together between 5 and 10 people.

The paper is organized as follows: In Section 2 we very briefly introduce ITSCM as described by the IT Infrastructure Library (ITIL) as well as SEAM. In Section 3 we explain the workshops we have run at FHVi and sketch the models created in these workshops. In Section 4 we discuss the findings and draw key learning points on service design. In Section 5 we briefly talk about related methods to SEAM. In Section 6 we summarize the work done and describe the next steps for this project.

\section{IT Service Continuity Management and SEAM}

ITSCM is defined in ITIL [9] as "The Process responsible for managing Risks that could seriously affect IT Services." ITIL further states that ITSCM depends on a broader process called Business Continuity Management (BCM). ITIL [9] describes a broad process for ITSCM; based on the assumption that most organizations either do 
not have a BCM process or that it is not really focused. This broad ITSCM process comprises the following activities:

- Initiation (Policy Setting, reference terms and scope, resource allocation, project organization, project and quality planning)

- Requirements and strategy (Business Impact Analysis, risk analysis, IT service continuity strategy)

- Implementation (Recovery planning, testing)

In ITIL, the Initiation and Requirements activities are part of BCM. ITSCM is only involved in these activities if they have not been done at all or not in enough detail so as to permit a good enough ITSCM.

Two of the main issues to be defined in BCM are called the Recovery Point Objective and the Recovery Time Objective. The Recovery Point Objective defines "the point [in time] to which data will be restored after recovery of an IT Service." [10]. This defines in essence the maximum amount of hours of operation in which data that can be lost. [10] gives the example that with daily backups up to 24 hours of data can be lost. The Recovery Time Objective "describes the maximum time allowed for recovery of an IT service following an interruption. The Service Level to be provided may be less than normal Service Level Targets." [10].

Most of the work in this project consisted in understanding how the application managers, and infrastructure managers were architecting their systems and to what extent this architecture was compliant with the requirements of psico. One important result was a refined Recovery Time Objective. It turned out to be a fairly complicated curve, which reflects the architectural considerations (what can be implemented or not by technology).

SEAM [15,16] is an Enterprise Architecture method designed from the ground up to model services and their implementation. SEAM was applied to the analysis and design of services in e.g. $[2,3,13]$. A SEAM model is typically made of several organizational levels, beginning with an organization providing a service to a set of beneficiaries. At this top-most organizational level, the organization is modeled as a whole (a black box) with just the service it offers visible to its beneficiaries. In the next organizational level the organization is viewed as a composite (white box). This level shows how the service is implemented. A succession of black boxes and white boxes can be represented until an organizational level that is meaningful to the stakeholders is reached. Through this hierarchy of service specifications and implementations, it is possible to relate the stakeholder's views. In this paper, the SEAM model spans from the representation of the service provided by a hospital to the general population down to the virtual machines, storage and network infrastructure. SEAM is based on a simplified UML representation in which only a handful of concepts have been kept (e.g. a class, an activity, a relationship). We augment these concepts with pictograms that represent systems (e.g. boxed arrow, UML components, UML stickman, etc.). The same concepts are used across all organizational levels to model the service offering and service implementation. Thus, the modelers only need to learn these very few graphical representations to model business and IT systems. This uniform representation also helps to validate whether the business and the IT are aligned. SEAM has a specific way of modeling organizations as service systems. A service system is said in [15] to "make use of its own resources and the resources of others to improve its circumstance and that of others." 
In the next section we describe the workshops and the models that were created including the way service systems are modeled with SEAM.

\section{Workshops and Models}

As mentioned in the introduction, the seven workshops that were run during this project were divided in two parts: The project scoping and the analysis.

\subsection{Project Scoping Workshops}

The project scoping workshops were run with the head of FHVi, his direct reports and the key people who were foreseen as project managers for this project to implement the continuity strategy. The three scoping workshops took a total of 9 hours with approx. 9 people in each workshop. These workshops helped scoping the project and defining that should be part of the ITSCM project team.

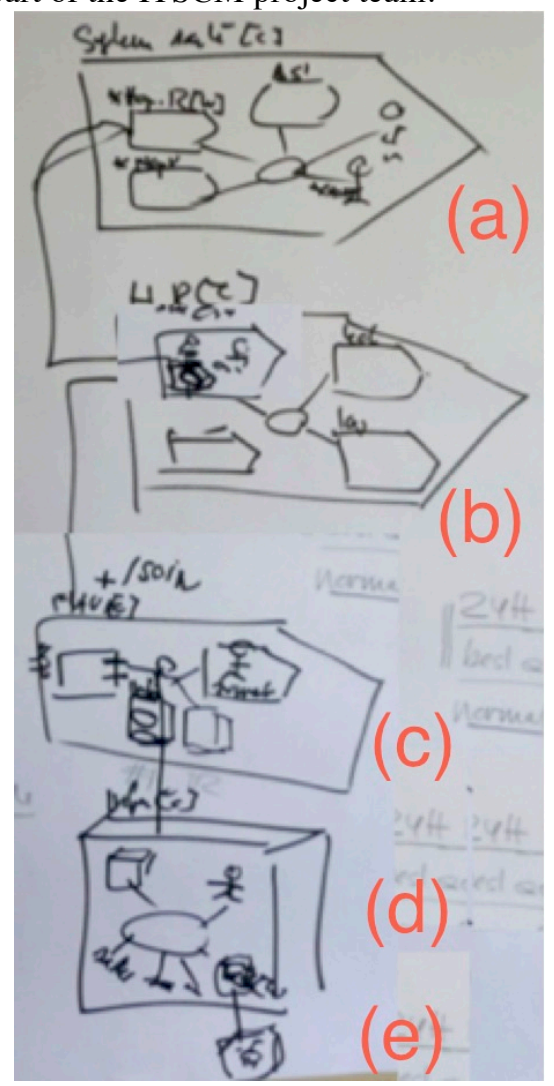

Figure 2: first sketch of service hierarchy (original workshop diagram): (a) service provided by hospital to population, (b) services provided by FHVi and 
doctors to implement hospital's service, (c) services of applications, help desk and infrastructure to implement FHVi 's services, (d and e) services provided by technical components to provide infrastructure services.

In the first workshop we developed a SEAM model with 4 organizational levels. Figure 2 illustrates the model sketched during the first workshop. We modeled the services provided at each level. These are:

- Services provided by an FHV hospital to the population (a in Figure 2),

- $\quad$ Services provided by FHVi and the medical staff to implement the services provided by the FHV hospital (b in Figure 2),

- Services provided by the applications and the infrastructure to implement the services provided by FHVi (c in Figure 2),

- Services provided by IT technical components to implement the services provided by the infrastructure ( $d$ and e in Figure 2).

The benefit of this session was to build a common understanding in the team of these levels of systems that implement services. This was crucial to divide responsibilities later on. After the first workshop, the model was cleaned up and drawn on a flipchart.

In the second workshop, this flipchart was used as canvas. The management team used post-it notes to populate the model. Yellow post-it notes represented existing elements. Pink post-it notes represented the change. The benefit of this workshop was a shared understanding of the project 'scope.

In the third workshop, the FHVi director and his staff agreed on the composition of the project team that will participate in the technical workshops, on deadlines for these workshops and on the deliverables. Figure 3 shows the model as developed during the second and third workshops. The model was then cleaned by the project leader and used to represent the project during the kick-off (March 2011).

\subsection{Technical Workshops}

The goal for the second series of workshops was to understand how to precisely relate the business requirements to the IT operations requirements, more precisely, the definition of the sequence in which the applications are to be resumed after the switch to the backup data center. We focused on the medical imaging service, which is considered to be one of the most complex services. The participants were mainly application managers and infrastructure managers. The manager of the psico project, the manager in charge of the backup center and the quality manager attended all workshops.

Four half-day workshops were run between May and July 2011. Overall the workshops lasted a total of approximately 12 hours with an average participation of 8 people.

The result of the four workshops is a model consisting of 4 organizational levels of services:

- FHVi and medical staff to implement services provided by FHV hospital,

- IT infrastructure and applications to implement FHVi services,

- Virtual machines and storage devices to implement IT infrastructure services, 
- Physical servers and OS to implement virtual machines services as well as network equipment to implement network services.

The first three levels are the same as those used in the scoping workshops. The last one was introduced in the technical workshops. These four levels were modeled three times, one for each operation mode, i.e. normal, disconnected, psico. The psico model shows what needed to be developed for the psico mode.

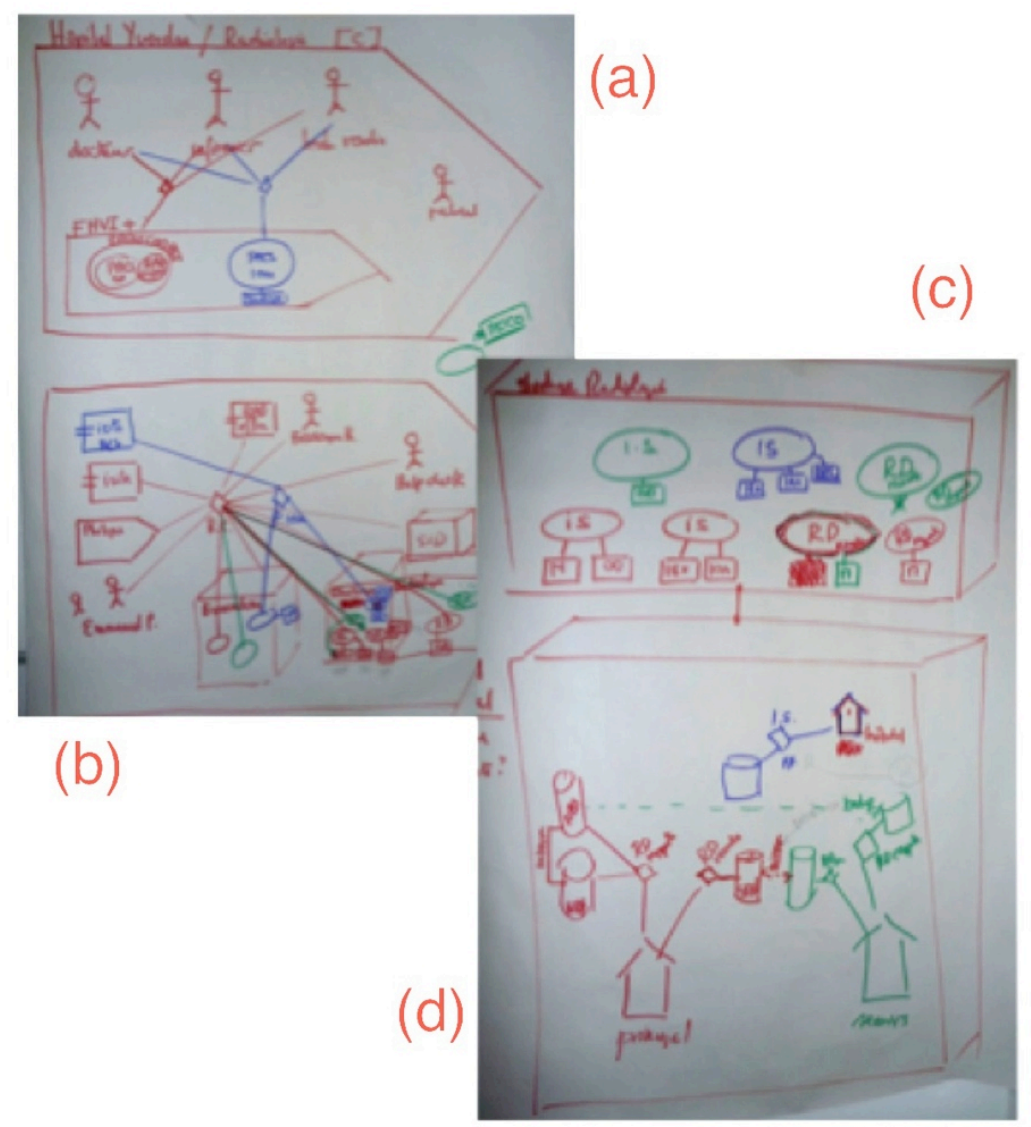

Figure 3: first technical workshop model (original workshop diagram). Part (a) describes the business processes and IT service. Part (b) describes the application and infrastructure services combined in IT processes. Part (c) describes the services provided by the storage infrastructure, it is a zoomed version of the bottom right model element in Part (b). Part (d) represents the implementation of the storage infrastructure.

The first technical workshop was held with 10 participants, mostly IT infrastructure people. We analyzed the sequence of transition between the normal, disconnected and reduced operation modes across the first three organizational levels. Figure 3 
illustrates this model. The part (a) of the diagram represents the service (ellipse in diagram) provided by FHVi to the doctors and how these services are combined in the business process (diamond in diagram). The color represents the modes (red $=$ normal, blue $=$ disconnected and green $=$ psico). In the diagram, $(b)$ represents how the services are implemented by combining applications and infrastructures in processes. Part (c) of Figure 3 represents the services provided by the storage infrastructure, it is actually a detail of the lower right elements in (b). Part (d) represents the technical processes inside the storage infrastructure.

The second technical workshop was held with application managers and a few infrastructure managers. During this meeting, we improved the models by better identifying the business roles of the service users. We also understood how the applications work together and the need to be able to access the medical and the administrative records in psico mode. We eventually added to the model, as a special kind of infrastructure, the middleware necessary to connect all these applications.

The third technical workshop was organized with the infrastructure managers. We made explicit that two broad kinds of storage techniques are used: databases in virtual machines, and dedicated storage devices SAN or NAS. The way data migrates between the main data center and the backup data center is done differently for each kind of storage technique. This leads to inconsistencies in the data after a switch to the backup data center. An architectural principle needs to be defined for the use of these storage techniques in a way that is compatible with the psico requirements. This also made us realize that the virtual machine strategy and the storage strategy could not be analyzed separately. This changed the structure of the SEAM models that were developed previously. The two separate systems: execution infrastructure and storage infrastructure had to be merged into one model element called storage and execution infrastructure.

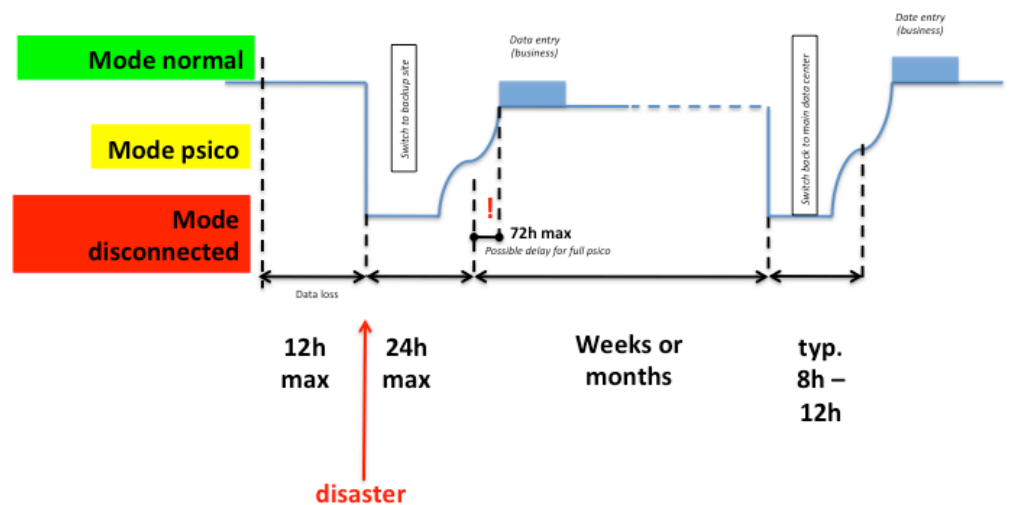

Figure 4: Diagram that illustrates the planned Recovery Time Objective

The last technical workshop brought together the application managers and the infrastructure managers for an overall review. We analyzed the time constraints related to the transition between operation modes: normal-disconnected, disconnectedreduced operation, reduced operation-normal. We made explicit that the levels of service was more complex than expected as shown in Figure 4. For example, the 
disconnected mode will also be used in the transition back from the backup site to the main data center (when returning to normal). We identified that data can be lost for up to 12 hours before the disaster occurrence (Recovery Point Objective). We also confirmed that the reduced mode of operation could last from several weeks to several months. This led us to decide that additional services (such computer assisted diagnostics) should be added to the reduced operation mode - even if they were not explicitly present in the list of critical services.

Overall we developed and reviewed 9 diagrams as illustrated in Figure 5: recovery time objective, 3 diagrams of FHVi / doctor level (green $=$ normal mode; yellow = psico mode; red = disconnected mode), 3 diagrams of application / infrastructure level, 1 diagram of storage and infrastructure (3 modes combined), 1 diagram of physical servers and 1 diagram of network devices.

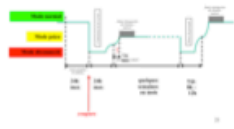

Métier (normal)

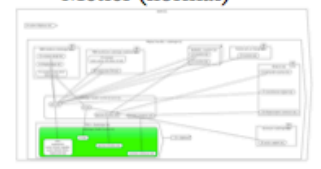

IT (normal)

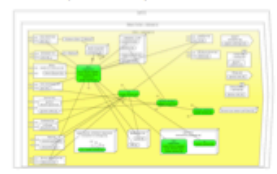

Résultats: profile, $3{ }^{*}$ métiers, 3 * IT, 1 * infra, $1{ }^{*} \mathrm{VM}, 1$ * réseau

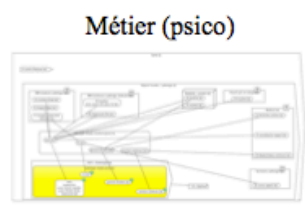

IT (psico)

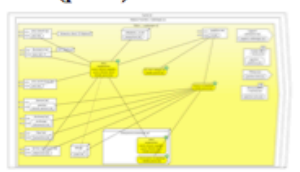

Métier (déconnecté)

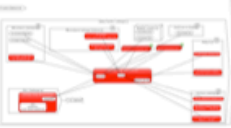

IT (déconnecté)

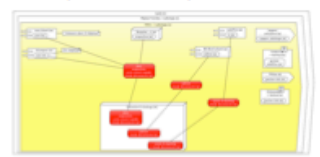

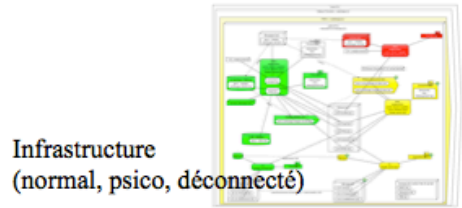

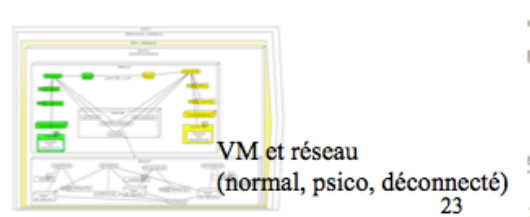

Figure 5: Bird's eye view of the models developed during the workshops (original cleaned-up diagrams)

We present in Figure 6 a simplified version of the models developed. It illustrates how SEAM models are built. This figure is drawn by hand because most models in the workshops were made by hand as well. This is important to give to the workshops participants the feeling that the diagrams capture work in progress. Otherwise, people tend to get locked in a "modeling stall" in a quest for the perfect model.

The service systems are visible in two organizational levels. For example, FHVi+ is represented as a whole in FHV hospital and as a composite in the next level. When a system is represented as a whole, its name is followed by "[w]", as composite by "[c]". When represented as a whole, a service system provides a service to its benefi- 
ciaries. When represented as a composite, we can model how the service is implemented with a process.

In the models, we use color to show the different modes: green $=$ Normal; yellow $=$ Psico and Red = disconnected. We also added the letters "N", "P", "D" for readers who have black and white pictures. These modes correspond to different functionalities (or number of functions provided). In the developed models, the functionality is described by properties (represented as rectangles) associated to the service. In the model, some elements are marked with "+". This indicates that this is an element to develop with the project.

The first organizational level shows the business roles that benefits from the services provided by $\mathrm{FHVi}+$. There is no difference visible between the three modes except that in the disconnected mode, paper has to be used for storing patient information instead of using the patient administrative management. In the real model, the details are visible (by showing the details of the services offered).

The second organizational level shows how, within FHVi+, the service is implemented by combining applications and infrastructure. We can also notice the help desk, and the supplier (S) that provides the applications. We represent the suppliers, as they are instrumental in providing the applications (FHVi outsources the development of applications). The "+" after the service system name (e.g. "FHVi+") reminds the workshop participants that the actors necessary to implement the service should be added to the system, even if they do not belong to the organization.

The third organizational level shows how the storage and execution infrastructure provides its service. We represent databases, buildings, a supplier and a virtual machine (VM) infrastructure. The buildings are marked " $\mathrm{H}$ " for hospital site, " $\mathrm{M}$ " for main site and " $\mathrm{B}$ " for backup site. There is a supplier that participates in the normal mode and the psico mode. In the normal mode, it acquires images for long-term image backup. In the psico mode, it provides access to the backup data and stores the new images generated. This illustrates that suppliers can be instrumental in providing a service and this is why they are visible in the diagram.

The last organizational level represents the VM system service that includes physical machines, the OS and the site in which the machines are located.

It is interesting to analyze how the concept of operation mode (normal, disconnected, psico) appears in each organizational level. In the upper level, services are clearly differentiated depending on the operation mode and location information is visible. In the execution and storage, the different modes cannot be as clearly separated. The implementations of the different mode use the same kind of infrastructure; locations become important. For example, the database stored in the hospital is used in the three modes. In the virtual machine level, location of the equipment is critical. This illustrates one of the challenges of working in business and IT alignment. Without understanding the evolution of the service offering between levels, it is very tempting to use implementation details to reference service levels. For example, before doing the workshops, it was common for the IT people to speak about the "backup site" when describing the service internally or to the business people. After the workshop, people talk about disconnected or psico mode. This is a better way to describe the service, as it is independent from the service implementation. 

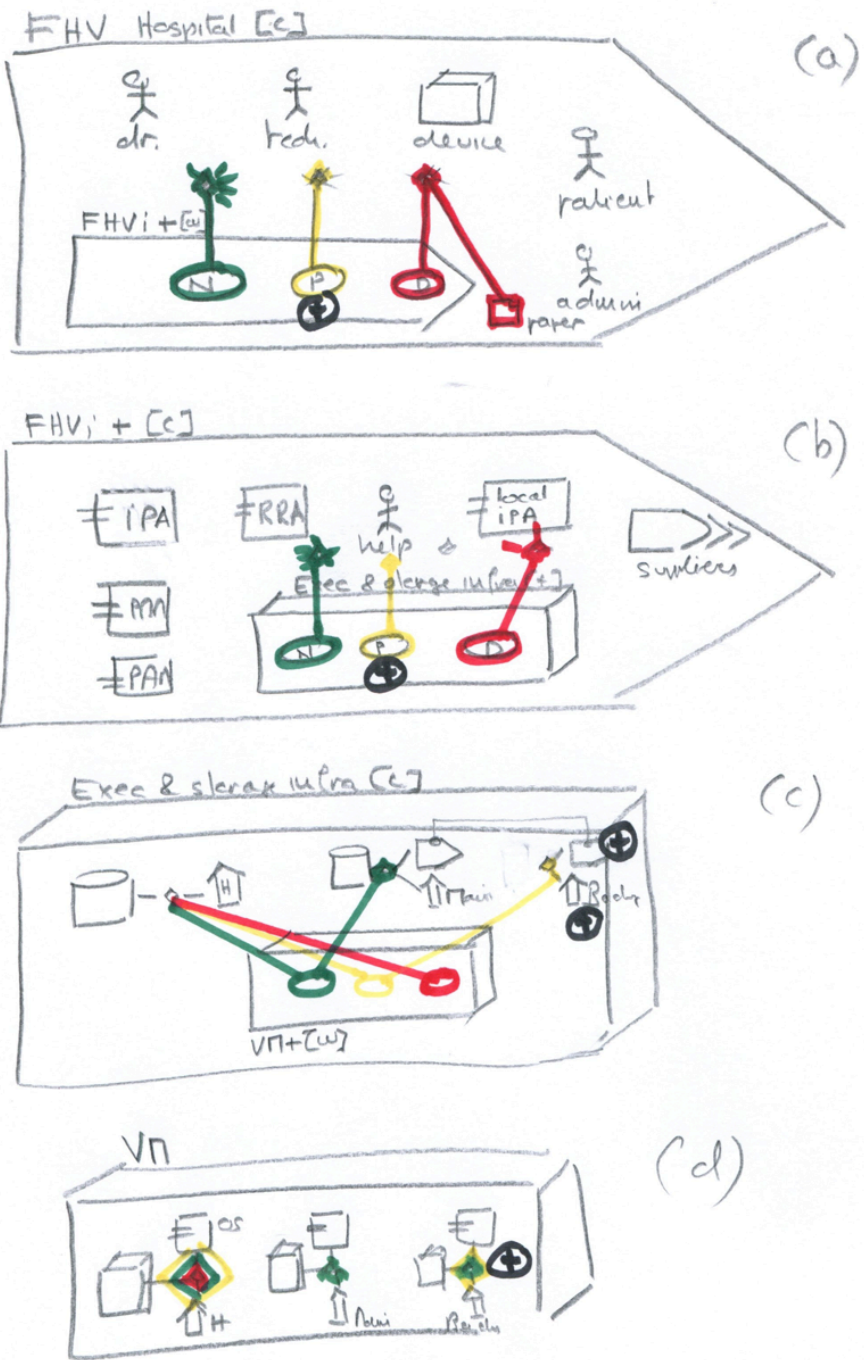

Figure 6: Structure of the SEAM model (simplified)

Once the models were developed, we had a wrap-up session to decide what needs to be done next. We decided that: (1) the models developed in the workshops will not be maintained and that the application documentation provided by the suppliers need to include some of this information, (2) an architecture group needs to be created to define the architectural principles for the IT systems. This group should issue a doc- 
ument that would capture these principles and the structuring assumptions concerning the IT systems. These assumptions are closely related to the way the SEAM model is structured (for example, merge of execution and storage infrastructure). The document will have an overview similar to Figure 6 (yet to be developed).

\section{Discussion}

This Section captures the findings and key learning points for the FHVi. They are broad enough to apply to any organization of similar scale. They are all based on the impact of a service view of the organization.

\subsection{Service oriented modeling to focus on delivery rather than structure}

It is always tempting in this kind of projects, to create models that follow organizational boundaries and reporting structures in which the modelers represent the organization as a suite of departments within departments. An important difference between a service model and an organizational model is the structuration as service systems as opposed to departments. A service system provides value to a stakeholder, which is outside the system. The beneficiary of what the department provides is less clearly identified and hence its value proposition is unclear. When we model service system, we have to include people, technology, suppliers, and any other resource necessary to implement the service within the system. Compare this with an organizational model, which usually doesn't include suppliers because they are not formally part of the organization. Also, in an organizational model, technology and people are often modeled separately and the department usually shows only the people because a department is related to a line of management.

\subsection{Service Oriented Analysis to Reduce Complexity}

Service models have several advantages:

- They minimize the impact of the organizational structure. People are grouped by service provided and not by line of management. This improves team spirit.

- The role of the suppliers is made highly visible. This is useful to make the organization and the supplier understand that the supplier is an instrumental component of the service supply.

- Potential conflicts of interests can be made visible. For example, a person might be inside a service system as provider of the service and outside the service system as beneficiary of the system.

- By focusing on a specific service, the complexity of the model is reduced as we can represent only one service at a time. 


\subsection{Service Oriented Analysis to Understand Architecture Requirements}

The initial question was "what in which sequence the services need to be resumed when switching from the main data center to the backup one". After analysis, it appears that this sequence is important for the IT people but not really for the business people. It also appeared that the people managing the virtual machines know how to resume the execution in the backup data center. They work with logical groups of virtual machines that need to be started in a given sequence (for example, network related VM such as DHCP servers need to start first). What appeared clearly is that this strategy works only if some key architectural principles, such as a consistent management of the data storage between applications, are respected (and communicated to all relevant parties - such as the suppliers). This makes visible the architecture challenges requirements that the organization needs to comply with.

The workshops also showed that one of the main challenges is the alignment between the applications and the IT infrastructure. IT infrastructure is evolving considerably with the new execution, storage and networking technologies. As a consequence, having an architecture that supports alignment between the applications and the infrastructure is key to guarantee the expected service levels. In the past, the alignment had to be made between business and IT. Now, it is between business and applications as well as between applications and infrastructure.

The workshop also captured significant clarification of the curve related to Recovery Time Objective, which were not available prior to the project. These clarifications are also direct consequences of the architectural choices taken.

\section{Related Work}

SEAM belongs to the Enterprise Architecture family. A distinct property of all these methods is their representation of organizations in hierarchical levels. Zachman founded the Enterprise Architecture discipline in the late 1980s with the so-called Zachman Framework [18]. The Zachman Framework and Urba-EA [5] are Enterprise Architecture methods that are used predominantly to model the landscape of the IT in a given organization. More recent Enterprise Architecture frameworks, e.g. TOGAF [14] and ArchiMate [4], encompass more the notion of service. ArchiMate models organizations and services as a succession of external and internal views [4]. Value is captured in the external view and implementation in the internal view.. ArchiMate has three levels of analysis, the business layer, Application layer and Technology layer. The SEAM models we have developed in this project span all these three layers. Just like SEAM, ArchiMate uses a subset of UML. The business layer, in most Enterprise Architecture methods, does not have the concepts necessary to model the service strategy of the organization with its stakeholders. An effort is under way [1] to augment ArchiMate with the Business Model Canvas [11] to fill this need.

The concept of service system was recently introduced in Service Science, e.g. in [8 and 15]. Early work in service system modeling is available in [6,7 and 12]. 


\section{Conclusions and Future Work}

In this paper we presented an on-going ITSCM project at an IT department that serves a federation of hospitals in the southwest part of Switzerland. The project is aimed at defining the service levels that the IT can provide to the hospitals in the event of a major incident (e.g. fire or flood) and aligning these levels with an appropriate architecture. We have shown the role played by service modeling with the SEAM Enterprise Architecture method in the understanding of these service levels and the resulting IT architecture. Now that the service modeling is completed, this project continues with the aim of harmonizing all the applications so that the overall service provided by the backup site conforms to the service levels defined previously.

\section{References}

1 Fritscher, B., Pigneur, Y.: Business IT Alignment from Business Model to Enterprise Architecture, Proc. Busital 2011, LNBIP 83, Springer (2011)

2 Golnam, A., Regev, G., Wegmann, A.: On Viable Service Systems: Developing a Modeling Framework for Analysis of Viability in Service Systems. In Snene, M., Ralyté, J., Morin, J.-H.,(Eds.): IESS 2011, LNBIP 82, pp. 30-41. (2011)

3 Golnam, A., Regev, G., Ramboz, J., Laprade, P., Wegmann, A.: Systemic Service Design: Aligning Value and Implementation. In Morin, J.-H., Ralyté, J., Snene, M. (Eds.): IESS 2010, LNBIP 53, pp. 150-164. (2010)

4 Lankhorst, M.M., Proper, H.A., Jonkers, H.: The Architecture of the ArchiMate Language. Proc. BPMDS 2009 and EMMSAD 2009, LNBIP 29, Springer (2009)

5 Longépé, C.: The Enterprise Architecture IT Project. KoganPage. (2003)

6 Kreuzer, E., Aschbacher, H.: Strategy-Based Service Business Development for Small and Medium Sized Enterprises. In Snene, M., Ralyté, J., Morin, J.-H.,(Eds.): IESS 2011, LNBIP 82, pp. 173-188. (2011)

7 Le Dinh, T., Pham Thi, T.T.: A Conceptual Framework for Service Modelling in a Network of Service Systems. In Morin, J.-H., Ralyté, J., Snene, M. (Eds.): IESS 2010, LNBIP 53, pp. 192-206. (2010)

8 Maglio, P.P., Vargo, S.L., Caswell, N., Spohrer, J., The service system is the basic abstraction of service science. Information Systems and E-Business Management 7:4, 395406 (2009)

9 Office of Government Commerce: ITIL Service Design, TSO, London, (2007)

10 Office of Government Commerce: ITIL Service Implementation, TSO, London, (2007)

11 Osterwalder, A., Pigneur, Y.: Business Model Generation. Self published (2010)

12 Poels, G.: A Conceptual Model of Service Exchange in Service-Dominant Logic. In Morin, J.-H., Ralyté, J., Snene, M. (Eds.): IESS 2010, LNBIP 53, pp. 224-238. (2010)

13 Regev, G., Hayard, O., Wegmann, A.: Service Systems and Value Modeling from an Appreciative System Perspective. In Snene, M., Ralyté, J., Morin, J.-H.,(Eds.): IESS 2011, LNBIP 82, pp. 146-157. (2011)

14 The Open Group Architecture Framework (TOGAF): http://www.opengroup.org/togaf: Accessed September 2011

15 Vargo, S.L., Maglio, P.P., Akaka, M.A.: On value and value co-creation: A service systems and service logic perspective. European Management Journal 26:3, 145-152, (2008)

16 Wegmann, A.: On the Systemic Enterprise Architecture Methodology (SEAM), Proc. International Conference on Enterprise Information Systems (ICEIS), Angers, France, (2003) 
17 Wegmann, A., Regev, G., Rychkova, I., Lê, L-S., De La Cruz, J.D., Julia, P.: Business-IT Alignment with SEAM for Enterprise Architecture. In: 11th IEEE International EDOC Conference, IEEE Press (2007)

18 Zachman, J. A.: A Framework for Information System Architecture. IBM Systems Journal 26(3): 276- 292, (1987) 